\title{
Lernen im Lebenslauf - Das lernende Subjekt im Spannungsfeld bildungspolitischer Imperative, pädagogischer Praxis und biografischer Forschung
}

\author{
Daniela Rothe
}

Online publiziert: 26. März 2015

(C) Die Autor(en) 2015. Dieser Artikel ist auf Springerlink.com mit Open Access verfügbar.

Zusammenfassung Im Kontext Lebenslangen Lernens werden Erwachsene zunehmend in einer biografischen Perspektive betrachtet. Der Artikel arbeitet heraus, wie unterschiedlich und zum Teil widersprüchlich Erwachsene als biografische Subjekte in der Bildungspolitik, in der Bildungspraxis und in der Erwachsenenbildungsforschung konstruiert werden. Es wird argumentiert, dass erst eine Verknüpfung von Biografieforschung und ethnografischen Methoden es ermöglicht $\mathrm{zu}$ untersuchen, welche Prozesse und Praktiken der biografischen Subjektivierung in der Erwachsenen- und Weiterbildung stattfinden und inwiefern diese dazu führt, dass Erwachsene sich selbst als biografische Lernsubjekte verstehen.

Schlüsselwörter Biografie $\cdot$ Biografieorientierung $\cdot$ Biografische Arbeit $\cdot$ Biografieforschung $\cdot$ Subjektivierung $\cdot$ Subjektorientierung $\cdot$ Lebenslanges Lernen

\begin{abstract}
In the context of lifelong learning, adults are increasingly being viewed from a biographical perspective. This article takes a look at how differently - and in some cases inconsistently - adults are construed as biographical subjects in education policy, in educational practice, and in research on adult education. It argues that to even begin to examine the processes and practices of biographical subjectification that take place in adult and continuing education, biographical research must be combined with ethnographic methods.
\end{abstract}

Keywords biography $\cdot$ biographical focus/orientation - biographical work $\cdot$ life history research $\cdot$ subjectification $\cdot$ subject orientation $\cdot$ lifelong learning

Dr. D. Rothe $(\triangle)$

Universität Wien, Wien, Österreich

E-Mail: daniela.rothe@univie.ac.at 


\section{Einleitung}

Mit der Etablierung der Rede vom Lebenslangen Lernen ist es üblich geworden, Erwachsene nicht nur situativ als Lernende - beispielsweise als Teilnehmende an einem Bildungsangebot - zu betrachten, sondern sie in einer lebenszeitlichen Perspektive mit ihren Lern- und Bildungsbiografien in den Blick zu nehmen und sie als biografische Subjekte anzusehen. Dies gilt nicht mehr nur für den Teil der Erwachsenenbildungsforschung, der mit den Mitteln der Biografieforschung die Bedeutung des Lernens in der Lebensspanne untersucht und die Bildungskontexte, die Teilnehmende als Lernende mit biografischen Erfahrungen adressieren, sondern auch für die Bildungspolitik, die seit Mitte der 1990er Jahre nachdrücklich versucht, Lernen als Imperativ der Lebensführung im Anschluss an die erste Bildungsphase durchzusetzen. Die Politik des Lebenslangen Lernens hat zweifellos die biografische Perspektive in der Erwachsenenbildung gestärkt - das gilt nicht nur für die Forschung (z. B. Alheit und Dausien 2002), sondern auch für bestimmte Bereiche der Bildungspraxis (z. B. Dausien 2003). Im Zuge dieser Entwicklung ist ein intensiviertes Interesse an den biografischen Subjekten zu beobachten: Diese werden im Rahmen von narrativ-biografischen Interviews aufgefordert, ihre Lebensgeschichte zu erzählen, die sie nicht selten selbst schon als Lern- und Bildungsgeschichte präsentieren. Spätestens in der Analyse der autobiografischen Selbstdarstellungen wird nach der expliziten und impliziten Bedeutung von Lernen und Bildung gefragt, nach den lebensgeschichtlichen Mustern in Lern- und Bildungsaktivitäten und nach den Kontinuitäten bzw. Diskontinuitäten, die darin erkennbar werden. Die Bildungspraxis begegnet den biografischen Hintergründen der Teilnehmenden und Adressaten mit neuer Aufmerksamkeit, sucht ihre Angebote auf sie abzustimmen und eröffnet Räume, in denen biografische Erfahrungen und biografisches Wissen thematisiert, expliziert und reflektiert werden können. Was in diesen verschiedenen Kontexten „biografisches Subjekt" heißt, steht im Mittelpunkt dieses Beitrags.

Subjekt, Subjektorientierung und Subjektivität sind als Begriffe in der Erwachsenenbildung seit den 1970er Jahren von Bedeutung, verweisen jedoch auf ganz unterschiedliche Theorietraditionen und Subjektmodelle. Man kann das Interesse an den Biografien der Lernenden als eine Variante von Subjektorientierung verstehen und darin neue Spielräume für individuelle Selbstbestimmung erkennen. Die zunehmende Rezeption poststrukturalistischer Zugänge (Moebius und Reckwitz 2008) in der Erziehungswissenschaft, die auch die Erwachsenenbildung erreicht hat (z. B. Wrana 2006), verweist jedoch auf die Ambivalenz dieser Entwicklung. Mit der Kritik am Subjektbegriff werden nicht nur die Grenzen der Möglichkeiten autonomer, handlungsmächtiger Subjekte, die über sich selbst verfügen können, aufgezeigt, sondern der Blick richtet sich auf die subtilen Prozesse der Reproduktion von gesellschaftlichen Machtverhältnissen gerade dort, wo Begriffe wie „Autonomie“ und „Selbstbestimmung“ besonders präsent sind. ${ }^{1}$ Subjektbildung bzw. Subjektivierung stellt sich - beispielsweise im Anschluss an Foucault (1992) - als ein Prozess dar, der

\footnotetext{
${ }^{1}$ Mit diesem Zusammenhang beschäftigen sich in der Erwachsenenbildung insbesondere Studien, die im Anschluss an Foucault das Konzept der Gouvernementalität nutzen, um gegenwärtige gesellschaftliche Lernverhältnisse zu beschreiben.
} 
gleichzeitig von Unterwerfung und Überschreitung bestimmt ist und die Grundlage von Handlungsfähigkeit bildet (Rieger-Ladich 2004). Diskursive Wissensordnungen der Pädagogik, der Politik, der Wissenschaft und die darin eröffneten Subjektpositionen stellen den Rahmen her, in dem sich Menschen als Subjekte begreifen können. Daraus sind eine Reihe neuer Begrifflichkeiten hervorgegangen wie Subjektivierung, Subjektivierungspraktiken oder Subjektformen (Reckwitz 2008), die auch für die Forschung über Lern- und Bildungsprozesse im Erwachsenenalter neue Fragestellungen aufwerfen.

Ausgehend von der Selbstverständlichkeit, mit der Erwachsene inzwischen als biografische Subjekte adressiert werden, werde ich in diesem Beitrag der Frage nachgehen, wie verschieden und ggf. auch widersprüchlich erwachsene Lernende als biografische Subjekte im Spannungsfeld zwischen Bildungspolitik, Bildungspraxis und Erwachsenenbildungsforschung konstruiert werden. Ich skizziere dafür zunächst die Bedingungen und Kontexte der biografischen Perspektive in der Erwachsenenbildungsforschung und -praxis (Kap. 2). Anschließend werde ich - unterschiedlich ausführlich - darauf eingehen, wie biografische Subjekte in der Bildungspolitik, Bildungspraxis und Forschung gegenwärtig (vor-)konstruiert werden. Dabei wird auch deutlich, wie sich Schwerpunktsetzungen des Interesses an der biografischen Dimension von Bildung im Erwachsenenalter verschoben haben (Kap. 3). AbschlieBend werde ich knapp darauf eingehen, inwiefern die Biografieforschung auch als ein empirischer Zugang zu Subjektivierungsprozessen im Kontext von Lernen und Bildung im Erwachsenenalter verstanden werden kann und welche (neuen) Zugänge und Fragestellungen damit verbunden sein können (Kap. 4).

\section{Bedingungen und Kontexte der Entwicklung der biografischen Perspektive in der Erwachsenenbildungsforschung und -praxis}

Die Themen, Fragestellungen und Konzepte in Bildungsforschung und Bildungspraxis werden nicht nur von konkreten Akteuren, beispielsweise Professionellen, Wissenschaftlerinnen und Wissenschaftlern, Administratorinnen und Administratoren entwickelt, sondern auch von den Bedingungen mitbestimmt, die sich aus dem Zusammenspiel von Bildungsforschung, Bildungspraxis und Bildungspolitik ergeben. Dies gilt auch für die biografische Perspektive. Drei dieser Bedingungen möchte ich grob skizzieren.

Die erste Bedingung betrifft das Verhältnis zwischen Erwachsenenbildungsforschung und Erwachsenenbildungspraxis. Historisch gesehen war Erwachsenenbildungsforschung über Jahrzehnte in der Bildungspraxis und nicht im universitären Kontext verankert und wurde von den Erwachsenenbildnern selbst durchgeführt (Zeuner und Faulstich 2009). Erst in den 1970er Jahren im Zuge der Bildungsreform wird die Erwachsenenbildung als Studien- und Forschungsschwerpunkt in der Erziehungswissenschaft universitär verankert. Der Praxisbezug bleibt zunächst bestehen, so dass auch von einer professionsbezogenen Wissenschaft gesprochen wird. In den vergangenen 20 Jahren sind empirische Forschung und Theoriebildung systematisch vorangetrieben worden, was zu einer Entkopplung von Erwachsenenbildungsforschung und -praxis beigetragen hat. Praxisbezüge in Form von Kooperationen, 
Entwicklungsprojekten, Praxisforschung, Evaluationen, Tagungen, Fortbildungen etc. bestehen jedoch nach wie vor und tragen zur Produktivität und Veränderung in Forschung und Praxis bei. Sie werden durch Förderstrukturen wie Forschungs- und Entwicklungsprogramme unterstützt. Das verweist bereits auf die zweite Bedingung.

Der bildungspolitische Kontext bestimmt die Dynamik der Erwachsenen-/Weiterbildung wesentlich mit - nicht in einer deterministischen Form, sondern in einem komplexen Wechselverhältnis. Das gilt für Einrichtung und Expansion des Fachs an der Hochschule im Zuge der Bildungsreform in den 1970er Jahren ebenso wie für den Einfluss der Bildungspolitik der Europäischen Union seit den 1990er Jahren. Politischer Einfluss wird nicht allein auf die Bildungspraxis genommen, sondern über Formen der Forschungs- und Entwicklungsförderung auch auf die Forschung. Da es um Altersgruppen geht, für die Erwerbstätigkeit von zentraler Relevanz ist, handelt es sich um Bildungspolitik, die gleichzeitig auch Wirtschafts-, Arbeitsmarktund Sozialpolitik ist, was an der Kofinanzierung einiger Programme durch den Europäischen Sozialfonds deutlich wird. Der Politik gelingt es auf diese Weise über die Ausschreibung von Förderlinien, Forschungsthemen zu setzen und zum Teil auch ihre Bearbeitungsrichtung mitzubestimmen. Darüber hinaus handelt es sich um ein Feld, in dem in hohem Maße symbolische Politik (Edelmann 2005 [1976]; Kuhlenkamp 1999) betrieben wird, d. h. der Raum, den das öffentliche Sprechen über Bedeutung und Notwendigkeit von Lernen und Bildung im Lebensverlauf einnimmt, ist mit zum Teil gegenläufigen strukturellen Entwicklungen im Feld der Erwachsenen-/Weiterbildung verbunden. Das betrifft insbesondere den öffentlich geförderten Bereich, der - parallel zu den bildungspolitischen Diskursen über die wachsende Bedeutung Lebenslangen Lernens - von Deckelung und Kürzung der öffentlichen (Grund-)Finanzierung sowie zunehmend kurzfristiger projektförmiger Bewilligung von Geldern bestimmt ist, von der freie Bildungsträger, Volkshochschulen und die berufliche Weiterbildung, die von der Bundesagentur für Arbeit (BA) gefördert wird, besonders betroffen sind (Nuissl und Brandt 2009, S. 35 f.). Daraus ergeben sich unmittelbare Folgen für den quartären Bereich.

Ich möchte an dieser Stelle auf zwei Veränderungen in der Erwachsenen- und Weiterbildungslandschaft verweisen. Mit dem Ziel, die Weiterbildung als vierte Säule des Bildungswesens auszubauen, war in den 1970er Jahren zunächst eine erhebliche Expansion dieses Bildungsbereichs zu beobachten. Bereits in den 1980er Jahren beginnt eine Phase der Stagnation bzw. des Rückbaus, der durch den im gleichen Zeitraum gestiegenen Anteil der Teilnehmerbeiträge gebremst wird. Diese Entwicklung wird in den 1990er Jahren teilweise verdeckt durch die Programme und Investitionen, die in dieser Zeit für den Auf- bzw. Umbau der Erwachsenen-/Weiterbildung in den neuen Bundesländern notwendig waren. Auch wenn die vierte „Säule“ des Bildungswesens etabliert ist, ist dieser Bereich vor allem marktförmig strukturiert. Probleme in der Zugänglichkeit für bildungsbenachteiligte sowie finanzschwache Gruppen bestehen nach wie vor, die kompensatorischen Effekte von Weiterbildung sind gering bzw. nicht vorhanden. Teilnahmestatistiken haben 2010 eine sinkende Beteiligung an Weiterbildung gezeigt. ${ }^{2}$ Reagiert wurde darauf vor allem mit dem

\footnotetext{
${ }^{2}$ Inzwischen sind die Teilnahmequoten wieder gestiegen, die Zuwächse im Bereich der betrieblichen Bildung fallen am größten aus (BMBF 2013).
} 
Versuch, die Teilnahmequoten über Kampagnen und finanzielle Anreize in Form von individuellen Zuschüssen (z. B. Bildungsprämien, Bildungssparen) zu erhöhen. Auch die Struktur der Erwachsenen- und Weiterbildungsforschung an den Universitäten hat sich seit der Etablierung des Fachs in den 1970er Jahren verändert. Bereiche der Erwachsenenbildung und außerschulischen Bildung wurden inzwischen wieder verkleinert, aufgelöst oder mit Bereichen beruflicher Weiterbildung zusammengelegt. Außeruniversitäre Forschungs- und Entwicklungseinrichtungen sind von diesen Strukturveränderungen ebenfalls betroffen.

\section{Die Konstruktion biografischer Subjekte in Bildungspolitik, Bildungspraxis und Erwachsenenbildungsforschung}

\subsection{Die bildungspolitische Perspektive auf das biografische Subjekt:}

Lernbereitschaft im Lebenslauf

In der Wiederbelebung des Konzepts Lebenslangen Lernens als bildungspolitisches Leitkonzept Mitte der 1990er Jahre spielen die Begriffe „Biografie“ und „Lebenslauf" keine Rolle, obwohl der biografische Ansatz in der Erwachsenenbildungsforschung etabliert und in Teilen der Bildungspraxis bereits verankert war. Es dauert fast zehn Jahre, bis die Begriffe Biografie und Lebenslauf in den bildungspolitischen Strategiepapieren sowie Forschungs- und Entwicklungsprogrammen einen Platz erhalten und die Rede von Lern- und Bildungsbiografien üblich wird. Nur mit erheblicher Verzögerung und sehr selektiv schließt der bildungspolitische Diskurs hier also an die fachwissenschaftliche Debatte an.

Analysen bildungspolitischer Dokumente zeigen, dass die sozialwissenschaftlichen Konzepte im Prozess der Rezeption und Einfügung in den bildungspolitischen Diskurs und seine Logik mit anderen Konzepten verschränkt werden und sich dabei inhaltlich verändern. ${ }^{3}$

Exemplarisch stehen dafür das vom Aktionsrat Bildung vorgelegte Reformkonzept „Bildung neu denken!“‘ (2003) und die von der Bund-Länder-Kommission für Bildungsplanung und Forschungsförderung (2004) vorgelegte Strategie des Lebenslangen Lernens in der Bundesrepublik Deutschland. Beide entwickeln einen biografischen Ansatz. „Bildungsbiografie“ meint darin ein Modell des Lebenslaufs, in dem fünf Phasen unterschieden werden: Kindheit, Jugend, junges Erwachsenenalter, Erwachsenenalter und Alter. Die Phasen verweisen auf eine Mischung aus lern- und entwicklungspsychologischen Modellen und dem eher soziologischen erwerbsarbeitszentrierten Lebenslaufmodell. Die Lebensaltersphasen treten als strukturierendes Prinzip an die Stelle der Bildungsinstitutionen, die nur noch als institutionelle Randbedingungen für das Lernen betrachtet werden, das auch jenseits konkreter Bildungseinrichtungen stattfinden kann und soll, worauf die besonders prominent gewordenen Konzepte selbstorganisierten und selbstgesteuerten Lernens verweisen. Im Strategiepapier der Bund-Länder-Kommission werden Entwicklungsschwerpunkte

\footnotetext{
${ }^{3}$ Für eine etwas genauere Rekonstruktion dieses Prozesses, die im Rahmen einer Studie über die diskursive Formation Lebenslanges Lernen entstanden ist, vgl. Rothe $2011 \mathrm{~b}$.
} 
wie z. B. Kompetenzentwicklung, Selbststeuerung und Lernberatung altersbezogen expliziert und bilden die Grundlage für bildungspolitisch initiierte Programme, Projekte und Maßnahmen, die Menschen in bestimmten Lebensaltersphasen adressieren. Die Pluralisierung und Ungleichheit der Lebensformen und -lagen, der biografischen Entwürfe und Verläufe, die in der Begründung der Programmatik Lebenslangen Lernens eine so wichtige Rolle spielen, erfahren hier eine bemerkenswerte Ausblendung. Auch wenn damit noch nicht gezeigt ist, ob und in welcher Weise dieses Modell praktische Wirksamkeit in der Organisation von Lern- und Bildungsangeboten im Erwachsenenalter entfaltet, wird sein normierendes Potenzial sichtbar. Die gerade für die biografietheoretische Perspektive auf Lernen so bedeutsamen Vorstellungen wie die biografische Eigenlogik von Prozessen der Sinn- und Erfahrungsbildung, die Relevanz sozialer und historischer Kontexte, in denen Lernen stattfindet, die komplexe und widersprüchliche Überlagerung unterschiedlicher individueller, institutioneller und gesellschaftlicher Zeitlogiken sowie die unterschiedlichen Ebenen der Reflexivität von Lernprozessen (Dausien 2008) sind in der bildungspolitischen Variante des biografischen Ansatzes nicht enthalten. ${ }^{4}$

Gleichwohl - und darin liegt die Funktionalität des Biografiebegriffs im bildungspolitischen Diskurs - erlaubt die Rede von der Lern- und Bildungsbiografie, das politisch eingeforderte kontinuierliche Lernen in der Lebensspanne auf einen positiven Begriff zu bringen. Insbesondere „Bildungsbiografie“ verweist weniger offensichtlich auf die Pädagogisierung des Erwachsenenalters, sondern eher auf eine bürgerliche Vorstellung von Bildung, die auf das ganze Leben und nicht nur die vorbereitenden Lebensphasen bezogen ist. Diese wird - so könnte man sagen - im Programm des Lebenslangen Lernens auf spezifische Weise demokratisiert: Jede und jeder kann und soll für sich in Anspruch nehmen, über seine bzw. ihre Bildungsbiografie zu sprechen und diese explizit zu gestalten. In der Diktion des bildungspolitischen Diskurses, der nicht an einen theoretisch bestimmten Bildungsbegriff anschließt, sondern ein Lernverständnis etabliert, das wesentlich an der anpassenden Bewältigung des gesellschaftlichen Wandels orientiert ist, müsste es konsequenterweise „Lernbiografie“ heißen. Die im bildungspolitischen Diskurs angelegte und präferierte Subjektform ist ein lernendes Subjekt, das sich selbst als Teil einer dynamischen Wissensgesellschaft und damit als permanent lernbedürftig und lernfähig versteht. Das schließt die Kultivierung lernförderlicher Dispositionen wie Lernbereitschaft und Motivation sowie entsprechender Kompetenzen zur Initiierung, Kontrolle und Evaluation der eigenen Lernprozesse ein (Rothe 2011a). Diese werden im institutionellen Rahmen des Bildungswesens eingeübt und verselbstständigen sich in einer Form von Selbstpädagogisierung.

Sieht man sich genauer an, welche Lernsubjekte im Fokus der Politik des Lebenslangen Lernens stehen, sind es vor allem gesellschaftliche Gruppen, die als ,,bildungsbenachteiligt“ und ,bildungsfern“ konstruiert werden - von Arbeitslosigkeit

\footnotetext{
${ }^{4}$ Das gegenwärtig auf nationaler Ebene relevante Strategiepapier ist mit „Konzeption der Bundesregierung zum Lernen im Lebenslauf“ (BMBF 2008) betitelt und beruht auf Empfehlungen des Innovationskreises Weiterbildung. Die zentralen Inhalte haben sich wenig verändert, treten aber eher pragmatisch, knapp und in dieser Form vor allem öffentlichkeitstauglich auf. Inwiefern der Lebenslaufbegriff hier überhaupt noch ein fachliches oder wissenschaftliches Konzept darstellt oder nur noch ein Alltagsbegriff ist, lässt sich kaum sagen.
} 
bedrohte Personen, Langzeitarbeitslose, Frauen (die nach der Familienphase in den Beruf zurückkehren), Migrantinnen und Migranten, Ältere und Menschen mit Behinderungen. Ihre Einbindung in kontinuierliche Lernprozesse ist vor allem daran orientiert, gesellschaftliche Probleme, wie z. B. soziale Exklusions- und Spaltungsprozesse, Arbeitslosigkeit und den demografischen Wandel zu verhindern bzw. zu bearbeiten. Gleichzeitig wird versucht, biografisch erworbene Ressourcen zugänglich und gesellschaftlich nutzbar zu machen, indem beispielsweise informell erworbene Kompetenzen sichtbar gemacht oder Wissen und Erfahrungen der Älteren, die in den Ruhestand übergehen, weitergegeben werden sollen.

\subsection{Das biografische Subjekt in der Bildungspraxis: Lernende als Experten für ihre} Lebensgeschichte

Spätestens seit den 1980er Jahren wird in der Erwachsenenbildungspraxis mit biografischen Ansätzen gearbeitet. Die Entwicklung dieser Ansätze ist eng mit den neuen sozialen Bewegungen verbunden, in denen sich Lernformen auch jenseits der institutionalisierten Erwachsenenbildung entwickeln. Die Frage nach dem Verhältnis zwischen Individuum und Gesellschaft spielt in den 1970er und 1980er Jahren nicht nur in sozialwissenschaftlichen Fachdebatten eine Rolle, sondern auch in den neuen sozialen Bewegungen und anderen individuellen und kollektiven Formen der Auseinandersetzung mit Identität, mit Erziehungsfragen, dem Geschlechterverhältnis oder der Rolle der älteren Generationen im Nationalsozialismus. In der Entwicklung biografischer Bildungsansätze wird selektiv auf die Biografieforschung, aber auch andere sozialwissenschaftliche und psychologische Theoriekonzepte (z. B. Oral History, Lebensweltforschung und Psychoanalyse, humanistische Psychologie) Bezug genommen. Insgesamt ist die Entwicklung biografieorientierter Ansätze in der Bildungspraxis jedoch nur punktuell mit der Biografieforschung in der Erwachsenenbildung verknüpft.

$\mathrm{Zu}$ den Bereichen, in denen sich im Laufe der Jahre biografische Ansätze etablieren konnten, zählen beispielsweise die historisch-politische Erwachsenenbildung (z. B. Behrens-Cobet und Reichling 1997; Behrens und Reichling 2011), die Altenbildung (z. B. Kade 2009; Ruhe 2009; Kulmus 2011), die Frauenbildungsarbeit (z. B. Haug 1990, 1999), die Alphabetisierungsarbeit (Fuchs-Brüninghoff et al. 1986; Arnold und Lucha 2011), aber auch die Professionalisierung in pädagogischen Berufen (z. B. Schulz 1996, 2008; Kirchhoff und Schulz 2008; Rothe 2008) und die Persönlichkeitsentwicklung (z. B. Gudjons et al. 1986, 2008). ${ }^{5}$ In der didaktischen Gestaltung in der allgemeinen Erwachsenenbildung sind Methoden verbreitet, die einen Bezug zu den biografischen Hintergründen der Teilnehmenden herstellen. Der kontinuierliche Erfolg von Methodensammlungen ist ein Indiz dafür. Die Art und Weise, aber auch die Intensität, in der in Bildungssituationen auf die Biografien der Teilnehmenden Bezug genommen wird, unterscheidet sich erheblich und reicht von eher punktuellen Methoden bis hin zur Unterstützung intensiver biografischer Selbst-

\footnotetext{
${ }^{5}$ Die hier angegebenen Quellen verweisen auf das Spektrum der für diesen Bereich typischen Literatur, die zwischen primär didaktisch orientierten Methodenbüchern und theoretisch begründeten Konzeptualisierungen angesiedelt ist. Wirklich systematische, bereichsübergreifende Darstellungen fehlen jedoch.
} 
reflexion. ${ }^{6}$ In der einen oder anderen Weise zielen alle diese Ansätze auf (Selbst-) Aufklärung über biografisches Geworden-Sein - unabhängig davon, ob dabei eher psychologische oder soziologische Modelle über das Verhältnis von Individuum und Gesellschaft leitend sind. Diese Aufgeklärtheit oder Reflexivität - so die explizite oder implizite Annahme - wird zur Voraussetzung für einen Gewinn an Selbstbestimmung der lernenden Subjekte in der Bildungssituation, beispielweise im Hinblick auf das Einbringen ihrer biografisch begründeten Interessen und Perspektiven. Der Zuwachs an Selbstbestimmung reicht im günstigen Fall über die Bildungssituation hinaus und eröffnet Handlungsspielräume im Alltag und für biografische Zukunftsperspektiven. Beide Momente sind in der Rede von der Expertenschaft für die eigene Lebensgeschichte enthalten. Es handelt sich also um eine Bildungsarbeit, in der die Teilnehmenden explizit an ihrem Selbst- und Weltverhältnis arbeiten. Das biografische Subjekt so zu denken und pädagogisch an seiner Hervorbringung zu arbeiten, verweist auf ein humanistisches Bildungs- und Subjektverständnis, ${ }^{7}$ d. h. ein Subjekt, dem Wissen und (Selbst-)Reflexion mehr Autonomie ermöglichen.

In den vergangenen $15 \mathrm{Jahren}$ haben sich außerdem neue Felder entwickelt, in denen explizit oder implizit auf ein biografisches Verständnis von Lernen im Lebenslauf rekurriert wird. Zu nennen sind so unterschiedliche Bereiche wie die Pflegebildung (z. B. Sander 2006), die Benachteiligtenförderung, die beispielsweise Jugendliche an den Übergängen zu Ausbildung und Erwerbsarbeit unterstützt (z. B. Raabe 2004), die (Weiter-)Bildungsberatung (Dausien 2011b), die sich verstärkt an den Lern- und Bildungsbiografien der Ratsuchenden orientiert. Biografieorientierte Kompetenzfeststellungsverfahren, wie die Arbeit mit dem ProfilPASS (Kollewe 2011), die jenseits formaler Bildungsabschlüsse und Zertifikate biografisch erworbene Kompetenzen ermitteln und sichtbar machen, um ihre Verwertbarkeit am Arbeitsmarkt zu ermöglichen, zählen ebenfalls dazu. Am anderen Ende des Qualifikationsspektrums in der beruflichen Weiterbildung weisen auch Konzepte wie Coaching oder Mentoring systematisch biografische Elemente auf. Selbstreflexion ist dabei mehr oder weniger explizit an der Optimierung der individuellen Arbeitskraft orientiert.

Gemeinsam ist den etablierten wie den neuen Zugängen zu den biografischen Erfahrungen der Teilnehmenden, dass sie in einer spezifischen - nämlich biografiebezogenen - Art und Weise die Aneignungsprozesse der Teilnehmenden zu unterstützen suchen. Sie setzen die Relevanz der biografischen Erfahrungen der Teilnehmenden nicht nur voraus, sondern wirken auf unterschiedliche Weise daran mit, dass sich die Teilnehmenden selbst als biografische Lernsubjekte verstehen, deren Lebensgeschichte ihr gegenwärtiges Lernen mitbestimmt und auf biografische Zukunftsentwürfe ausrichtet. Eine solche Praxis wirkt auch explizit daran mit, wie dieses Lernsubjekt ,aussieht“ - für andere und für sich selbst - insbesondere hinsichtlich seines Wissens, seiner Kompetenzen und seines Selbstverständnisses.

\footnotetext{
${ }^{6}$ Dausien (2011a, S. 116) unterscheidet zwischen Biografie als Hintergrund, Lernfeld und Gegenstand von Bildungsprozessen.

${ }^{7}$ Es ist naheliegend, dass diese Bildungsangebote besonders Personen ansprechen, die über vergleichsweise viel kulturelles Kapital verfügen, auch wenn die Arbeitsfelder zeigen, dass versucht wird, auch mit anderen Zielgruppen biografieorientiert zu arbeiten.
} 
Empirische Forschungen über die unterschiedlichen biografieorientierten Ansätze in der Erwachsenen- und Weiterbildungspraxis, über die Konzepte und die Instrumente, ihre Verbreitung und die Art und Weise, wie sie von Professionellen und Lernenden unter spezifischen institutionellen Bedingungen der Erwachsenen-/Weiterbildung gebraucht und genutzt werden, liegen bisher nicht vor. Etablierte und neue biografieorientierte Lern- und Bildungsarrangements sind Orte, an denen Teilnehmende und Adressaten nicht nur als biografische Subjekte angesprochen werden, sondern auch interaktiv gerahmte Prozesse der biografischen Arbeit und Subjektivierung stattfinden, d. h. Subjekte mit professioneller Unterstützung und unter Nutzung von Instrumenten der Selbstbefragung und Selbstreflexion sich selbst verändern.

\subsection{Das biografische Subjekt in der Erwachsenenbildungsforschung}

Die soziologische Biografieforschung wird in der Erwachsenenbildung in den 1980er Jahren rezipiert und bewegt sich zunächst zwischen interpretativer Biografieforschung (z. B. Alheit 1984a, b), Lebenslaufforschung (z. B. Siebert 1985) und Entwicklungs- und Sozialpsychologie (z. B. Knoll 1980). Die gerade erst universitär verankerte Teildisziplin ist etwa zeitgleich mit der theoretischen Bestimmung ihres zentralen Gegenstandes beschäftigt - nämlich der Frage, was ein Erwachsener eigentlich ist und was das für die Erwachsenenbildung bedeutet - und bedient sich dafür aus den Theorieangeboten der Nachbardisziplinen. Soziologische Konzepte wie Erwachsenensozialisation, Biografie, Lebenswelt und Lebenslauf und entwicklungspsychologische Modelle des Lebenszyklus, der Lebens(alters)phasen werden genutzt und nehmen Einfluss darauf, wie Lernprozesse und lernende Subjekte modelliert werden. Die Rezeption entwicklungspsychologischer Modelle und soziologischer Forschung ermöglicht es, von autonomen, erwachsenen Lernsubjekten auszugehen, aber gleichzeitig deutlich zu machen, dass Autonomie im Erwachsenenalter eingeschränkt ist bzw. an ihrem Erhalt - auch im Rahmen von (organisierten) Bildungsprozessen - immer wieder gearbeitet wird bzw. werden muss. Infrage gestellt wird autonome Handlungsfähigkeit - je nach theoretischen Präferenzen - entweder durch Identitätskrisen in der Bearbeitung von Entwicklungsaufgaben, durch kritische Lebensereignisse etc. oder durch gesellschaftliche Veränderungs- und Modernisierungsprozesse, die die Lebenslaufmuster verändern, Übergänge beispielsweise in Erwerbstätigkeit verzögern und damit ökonomische Eigenständigkeit verhindern. Enttraditionalisierung und Pluralisierung der Lebensformen erzeugen nicht nur neue Handlungsmöglichkeiten, sondern auch neue Zwänge, Abhängigkeiten und Handlungsunsicherheiten. Zeitdiagnostische Theorien wie die der reflexiven Modernisierung (vgl. Beck et al. 1996) bilden in der Folgezeit eine wichtige Begründung sowohl für biografische Forschung als auch für Bildungsbedarfe im Erwachsenenalter. Der Identitätsbegriff rückt im weiteren Verlauf in den Hintergrund. Stattdessen werden biografische Erfahrungsprozesse und Handlungsstrategien beispielsweise im Umgang mit Übergängen im Lebenslauf und in der Gestaltung unterschiedlicher Lebensphasen untersucht und gefragt, welche Lern- und Bildungsprozesse dabei stattfinden.

Der Anschluss an ein sozialwissenschaftliches Biografieverständnis ermöglicht es, forschungspraktisch eine defizitorientierte Sichtweise zu überwinden und erwach- 
sene Subjekte als kompetente Erzähler und Erzählerinnen ihrer Lebensgeschichte zu adressieren. So können biografische Subjekte, die im Kontext konkreter lebensweltlicher Einbindung und gesellschaftlicher Bedingungen Handlungsmöglichkeiten haben, einen Lebensentwurf mehr oder weniger eingeschränkt realisieren (Alheit und Dausien 2000). In der Rekonstruktion geht es immer auch darum, zu zeigen, wie Individualität und Gesellschaftlichkeit in biografischen Konstruktionen verschränkt sind. Mit der Rezeption der biografischen Perspektive findet also auch eine Verschiebung in der Konstruktion des lernenden Subjekts statt. ${ }^{8}$ Die in den 1970er und 1980er Jahren verbreitete Defizitorientierung im Blick auf den „,bildungsbedürftigen Erwachsenen" wird theoretisch und empirisch relativiert.

Institutionalisierte Bildungsangebote scheinen in vielen Lebensgeschichten gar keine Rolle zu spielen (Alheit 1984a, b). Gleichzeitig - und das macht einen Teil der Attraktivität des Zugangs aus - verbindet sich damit die Erwartung, biografische Erfahrungen und Lernprozesse außerhalb bzw. jenseits von Bildungsinstitutionen - z. B. im Kontext von Arbeit, politischem und sozialem Engagement und in der Lebenswelt bzw. im Alltag - der empirischen Untersuchung zugänglich zu machen. Allerdings zeigt sich - insbesondere auch in der biografischen Forschung, die durch die Wiederbelebung des Konzepts Lebenslangen Lernens angeregt worden ist, wie schwierig es ist, Lern- und Bildungsprozesse in lebensgeschichtlichen Erzählungen zu rekonstruieren. Für die Mehrheit dieser Forschung bildet die Teilnahme an formalisierten Lernarrangements bereits im Forschungsdesign einen wichtigen Bezugspunkt (z. B. Kade und Seitter 1996; Alheit et al. 2003; Justen 2011; Kade et al. 2008; Shell-Kiel 2007) und spielt vermutlich auch in der Interviewsituation und damit in der biografischen Selbstpräsentation eine Rolle. Die damit verbundene Fokussierung auf bildungsaffine Milieus ist möglicherweise eine Einschränkung, weil andere gesellschaftliche Gruppen und unter Umständen spezifische Muster der biografischen Subjektbildung in Lern- und Bildungsprozessen gar nicht erst in den Blick geraten.

Die in der Regel fallrekonstruktiv angelegten Untersuchungen zeigen, dass Lernprozesse und die Aneignung institutioneller Bildungsangebote im Erwachsenenalter biografischen Eigenlogiken folgen, die für die biografischen Subjekte jedoch nur begrenzt durchschaubar und deshalb auch nur bedingt verfügbar sind. Sie können aber in der Rekonstruktion und Analyse der Lebensgeschichten sichtbar gemacht werden. Das betrifft beispielsweise die zeithistorische Bedingtheit von Lernpraktiken und Bildungserfahrungen durch Diskurse und Wissensordnungen der Pädagogik, der Politik, der massenmedial vermittelten Öffentlichkeit, wie in den aktuellen Studien über die Kontextualität von Bildungsgestalten im Zeitvergleich (Kade und Nolda 2014a, b) empirisch deutlich wird. Im Kontext der Politik des Lebenslangen Lernens stellt sich die empirische Frage, welche Spuren die Anrufung als lernendes Subjekt in den Selbstverständnissen und biografischen Konstruktionen erwachsener Gesellschaftsmitglieder hinterlässt.

\footnotetext{
${ }^{8}$ Das ist natürlich auch eine Vereinfachung, weil das im sozialwissenschaftlichen Biografiebegriff potenziell enthaltene Subjektverständnis gerade auch im Hinblick auf Möglichkeiten von Autonomie unterschiedlich ausgelegt wird.
} 


\section{Ausblick: Praktiken und Prozesse der Biografisierung im Kontext Lebenslangen Lernens}

Was hat die Frage nach den verschiedenen Verständnissen des lernenden Subjekts in Bildungspolitik, Bildungspraxis und Erwachsenenbildungsforschung ergeben und welche Konsequenzen lassen sich daraus für die Biografieforschung in der Erwachsenenbildung ableiten? Im Vergleich werden eine Reihe von Differenzen und Ungleichzeitigkeiten erkennbar. So orientiert sich die Politik des Lebenslangen Lernens an Modellen, die in der Erwachsenenbildungsforschung überwunden sind und operiert einer politischen Logik entsprechend - mit einer Vorstellung vom Subjekt, das so disponiert ist, dass es selbstverantwortlich sein Lernen und seine Lernbiografie gestaltet. Die das Lernen im Erwachsenenalter bestimmende gesellschaftliche Ungleichheit bleibt als solche unthematisiert und wird vor allem an der gesteigerten Aufmerksamkeit für die Personengruppen erkennbar, die statistisch gesehen wenig oder gar nicht an Weiterbildung teilnehmen und deshalb als „,bildungsfern“ bezeichnet werden. Dabei wird auch eine eher individualisierende Form der Defizitorientierung wiederbelebt. Die Explizierung der Differenzen in den Subjektvorstellungen erzeugt an dieser Stelle eine Distanz zu bildungspolitischen Argumentationsfiguren, die von der Erwachsenenbildungsforschung produktiv genutzt werden kann, um gerade auch in einer biografischen Perspektive empirisch zu zeigen, wie die institutionellen Strukturen des Bildungswesens Bildungswege bestimmen und Lernen im Erwachsenenalter nicht nur ermöglichen, sondern auch behindern und unsichtbar machen. Eine rekonstruktiv angelegte Biografieforschung kann zeigen, wie zeitgeschichtliche Kontexte und sich verändernde pädagogische und bildungspolitische Wissensordnungen autobiografische Selbstpräsentationen von Lern- und Bildungsbiografien mitbestimmen. Dabei kann auch herausgearbeitet werden, inwiefern das Bildungswesen dabei zur Reproduktion gesellschaftlicher Ungleichheit beiträgt und unter welchen Bedingungen Bildung im Erwachsenenalter neue biografische Handlungsräume eröffnet.

Es ist außerdem deutlich geworden, dass im Kontext der Politik des Lebenslangen Lernens biografieorientierte Ansätze inzwischen auch in mehr Bereichen der Erwachsenen-/Weiterbildung genutzt werden. Neben den Ansätzen der politischen Bildung, die anhand biografischer Erfahrungen das Verhältnis von Subjekt und Gesellschaft zum Gegenstand der (Selbst-)Reflexion machen, etabliert sich Biografieorientierung auch in der Bildungsarbeit mit unterschiedlichen benachteiligten Gruppen beispielsweise an Übergängen im Lebenslauf. Diese Arbeit ist von ganz anderen institutionellen Bedingungen und Logiken der Verwertbarkeit bestimmt. Hier öffnet sich ein interessantes Feld für die Biografieforschung, insbesondere in der Verbindung mit ethnografischen Methoden und Interaktionsanalysen. Auf diese Weise kann untersucht werden, welche Praktiken der Biografisierung in pädagogischen Arrangements dazu beitragen, dass Menschen sich als biografische Lernsubjekte verstehen lernen. So können empirisch Prozesse der biografischen Subjektivierung analysiert werden, die im Kontext Lebenslangen Lernens in der konkreten Erwachsenen- und Weiterbildungspraxis stattfinden. Die theoretische Perspektive der Subjektivierung ermöglicht es dabei auch, zu fragen, wie diese Prozesse in institutionelle und gesellschaftliche Macht- und Ungleichheitsverhältnisse eingebunden sind und zu ihrer Reproduktion beitragen. 
Relevanz und Anschlussfähigkeit solcher Forschungen für die Bildungsarbeit mit Erwachsenen, die es immer auch mit den biografischen Erfahrungen und Hintergründen der Teilnehmenden zu tun hat und diese zuweilen auch methodisch explizit zum Gegenstand von Lernprozessen macht, besteht nicht in erster Linie in der Optimierung methodischer Zugänge. Vielmehr geht es darum, die biografische Dimension von Lern- und Bildungsprozessen im Erwachsenenalter besser zu verstehen, und auf dieser Grundlage Bildungssituationen so zu gestalten, dass auch eigensinnige biografische Prozesse möglich sind, die durchaus im Widerspruch zu den Steuerungsansprüchen stehen können, die gerade auch im Kontext Lebenslangen Lernen eine Renaissance erleben und mit Normierung und Disziplinierung der Lernenden verbunden sind. In welcher Form daraus neue wechselseitig produktive Bezüge zwischen Erwachsenenbildungspraxis und Biografieforschung entstehen können, ist dabei offen und bedarf expliziter Aufmerksamkeit.

Open Access Dieser Artikel unterliegt den Bedingungen der Creative Commons Attribution License. Dadurch sind die Nutzung, Verteilung, und Reproduktion erlaubt, sofern der/die Originalautor/en und die Quelle angegeben sind.

\section{Literatur}

Alheit, P. (1984a). Biographieforschung in der Erwachsenenbildung 1. Literatur- und Forschungsreport Weiterbildung, 13, 40-54.

Alheit, P. (1984b). Biographieforschung in der Erwachsenenbildung 2. Literatur- und Forschungsreport Weiterbildung, 14, 31-67.

Alheit, P., \& Dausien, B. (2000). Die biographische Konstruktion der Wirklichkeit. Überlegungen zur Biographizität des Sozialen. In E. Hoerning (Hrsg.), Biographische Sozialisation (S. 257-283). Stuttgart: Lucius \& Lucius.

Alheit, P., \& Dausien, B. (2002). Bildungsprozesse über die Lebensspanne und Lebenslanges Lernen. In R. Tippelt (Hrsg.), Handbuch Bildungsforschung (S. 565-585). Opladen: Leske + Budrich.

Alheit, P., Dausien, B., Kaiser, M., \& Truschkat, I. (2003). Neue Formen (selbst) organisierten Lernens im sozialen Umfeld. Qualitative Analyse biographischer Lernprozesse in innovativen Lernmilieus (QUEM-Materialien Bd. 43). Berlin: Arbeitsgemeinschaft Betriebliche Weiterbildungsforschung e. V.

Arnold, U., \& Lucha, D. (2011). „Biographisches Arbeiten in der Grundbildung“. Eine Fortbildung zur Professionalisierung von Kursleitenden. In B. Egloff \& A. Grotlüschen (Hrsg.), Forschen im Feld der Alphabetisierung und Grundbildung. Ein Werkstattbuch (S. 227-236). Münster: Waxmann.

Beck, U., Giddens, A., \& Lash, S. (1996). Reflexive Modernisierung. Eine Kontroverse. Frankfurt a. M.: Suhrkamp.

Behrens-Cobet, H., \& Reichling, N. (1997). Biographische Kommunikation: Lebensgeschichten im Repertoire der Erwachsenenbildung. Neuwied: Luchterhand.

Behrens, H., \& Reichling, N. (2011). Debattierende Vielfalt statt ,innere Einheit“. Biographische Ansätze in der deutsch-deutschen Bildungsarbeit seit 1990. Hessische Blätter für Volksbildung, 2, 135-142.

Dausien, B. (2003). Zielgruppen - Lebenswelten - Biographien. Sichtweisen der Erwachsenenbildung auf „die Teilnehmenden“. In P. Ciupke, B. Faulenbach, F. J. Jelich, \& N. Reichling (Hrsg.), Erwachsenenbildung und politische Kultur in Nordrhein-Westfalen. Themen - Institutionen - Entwicklungen seit 1945 (S. 31-50). Essen: Klartext.

Dausien, B. (2008). Lebenslanges Lernen als Leitlinie für die Bildungspraxis? Überlegungen zur pädagogischen Konstruktion von Lernen aus biographietheoretischer Sicht. In H. Herzberg (Hrsg.), Lebenslanges Lernen. Theoretische Perspektiven und empirische Befunde im Kontext der Erwachsenenbildun (S. 151-174). Frankfurt a. M.: Peter Lang.

Dausien, B. (2011a). „Biographisches Lernen“ und „Biographizität“. Überlegungen zu einer pädagogischen Idee und Praxis in der Erwachsenenbildung. Hessische Blätter für Volksbildung, 2, 110-125. 
Dausien, B. (2011b). „Das beratene Selbst“ - Anmerkungen zu Bildungsbiographien im gesellschaftlichen Wandel und Strategien ihrer professionellen Bearbeitung. In M. Hammerer, E. Kanelutti, \& I. Melter (Hrsg.), Zukunftsfeld Bildungs- und Berufsberatung. Neue Entwicklungen aus Wissenschaft und Praxis (S. 21-40). Bielefeld: W. Bertelsmann Verlag.

Edelmann, M. (2005) [1976]. Politik als Ritual. Die symbolische Funktion staatlicher Institutionen und politischen Handelns. Frankfurt a. M.: Campus.

Foucault, M. (1992). Was ist Kritik? Berlin: Merve Verlag.

Fuchs-Brünighoff, E., Kreft, W., \& Kropp, U. (1986). Alphabetisierung - Konzept und Erfahrungen. Bericht eines Projekts des Bundesministeriums für Bildung und Wissenschaft. Bonn: Deutscher Volkshochschul-Verband.

Gudjons, H., Wagener, B., \& Pieper, M. (1986). Auf meinen Spuren. Das Entdecken der eigenen Lebensgeschichte. Vorschläge und Übungen für pädagogische Arbeit und Selbsterfahrung. Reinbek bei Hamburg: Rowohlt Verlag.

Gudjons, H., Wagener-Gudjons, B., \& Pieper, M. (2008). Auf meinen Spuren. Übungen zur Biografiearbeit. Bad Heilbrunn: Klinkhardt.

Haug, F. (1990). Erinnerungsarbeit. Hamburg: Argument.

Haug, F. (1999). Vorlesungen zur Einführung in die Erinnerungsarbeit. The Duke Lectures. Hamburg: Argument.

Justen, N. (2011). Erwachsenenbildung in biographischer Perspektive. Lebensgeschichte - Bildungsmotive - Lernprozesse. Opladen: Barbara Budrich.

Kade, S. (2009). Altern und Bildung. Eine Einführung. Bielefeld: W. Bertelsmann Verlag.

Kade, J., \& Nolda, S. (2014a). 1984/2009 - Bildungsbiografische Gegenwart im Wandel von Kontextkonstellationen. Zeitschrift für Pädagogik, 60, 587-606.

Kade, J., \& Nolda, S. (2014b). Individualitätsperformanz. Bildungsbiographische Anspruchsindividualitäten in sich wandelnden Kontexten. In F. von Rosenberg \& A. Geimer (Hrsg.), Bildung unter Bedingungen kultureller Pluralität (S. 125-153). Wiesbaden: VS Verlag.

Kade, J., \& Seitter, W. (1996). Lebenslanges Lernen. Mögliche Bildungswelten. Erwachsenenbildung, Biographie und Alltag. Opladen: Leske + Budrich.

Kade, J., Hof, C., \& Peterhoff, D. (2008). Verzeitlichte Bildungsgestalten: Subjektbildung im Kontext des Lebenslangen Lernens. Report. Zeitschrift für Weiterbildungsforschung, 31, 9-22.

Kirchhof, S., \& Schulz, W. (Hrsg.). (2008). Biografisch lernen \& lehren. Möglichkeiten und Grenzen zur Entwicklung biografischer Kompetenz. Flensburg: Flensburg University Press.

Knoll, J. H. (1980). Lebenslauf, Lebenszyklen und Erwachsenenbildung. Internationales Jahrbuch der Erwachsenenbildung, 8, 159-185.

Kollewe, L. (2011). Die ProfilPASS-Beratung als biografischer Lernprozess im Spannungsfeld von Beratung und Therapie. Hessische Blätter für Volksbildung, 2, 161-168.

Kuhlenkamp, D. (1999). Tendenzen in der Gesetzesentwicklung auf Länderebene. In W. Gieske \& R. Arnold (Hrsg.), Die Weiterbildungsgesellschaft. Bd. 2. Bildungspolitische Konsequenzen (S. 158176). Neuwied: Luchterhand.

Kulmus, C. (2011). Perspektiven biografischer Arbeit mit Älteren. Hessische Blätter für Volksbildung, 2, 152-160.

Moebius, S., \& Reckwitz, A. (2008). Poststrukturalistische Sozialwissenschaften. Frankfurt a. M.: Suhrkamp.

Nuissl, E., \& Brandt, P. (2009). Porträt Weiterbildung Deutschland. Bielefeld: W. Bertelsmann Verlag.

Raabe, W. (2004). Biografiearbeit in der Benachteiligtenförderung. Heidelberg: Hiba.

Reckwitz, A. (2008). Subjekt/Identität. Die Produktion und Subversion des Individuums. In S. Moebius \& A. Reckwitz (Hrsg.), Poststrukturalistische Sozialwissenschaften (S. 75-92). Frankfurt a. M.: Suhrkamp.

Rieger-Ladich, M. (2004). Unterwerfung und Überschreitung: Michel Foucaults Theorie der Subjektivierung. In N. Ricken \& M. Rieger-Ladich (Hrsg.), Michel Foucault. Pädagogische Lektüren (S. 203223). Wiesbaden: VS Verlag.

Rothe, D. (2008). Pädagogische Biographiearbeit. Ein Wissenschaft-Praxis-Projekt zu Professionalisierung pädagogischen Handelns. In S. Kirchhof \& W. Schulz (Hrsg.), Biografisch lernen \& lehren. Möglichkeiten und Grenzen zur Entwicklung biografischer Kompetenz (S. 147-167). Flensburg: Flensburg University Press.

Rothe, D. (2011a). Lebenslanges Lernen als Programm. Eine diskursive Formation in der Erwachsenenbildung. Frankfurt a. M.: Campus. 
Rothe, D. (2011b). Zwischen Bildungsbiographie und Lernen im Lebenslauf: Konstruktionen des Biographischen in der Politik des Lebenslangen Lernens. In H. Herzberg \& E. Kammler (Hrsg.), Biographie und Gesellschaft. Überlegungen zu einer Theorie des modernen Selbst (S. 43-59). Frankfurt a. M.: Campus.

Ruhe, H. G. (2009). Methoden der Biografiearbeit. Lebensspuren entdecken und verstehen. Weinheim: Juventa.

Sander, K. (2006). Biografiearbeit. Grundlagen der Pflege für die Aus-, Fort- und Weiterbildung 21. Brake: Prodos Verlag.

Schell-Kiehl, I. (2007). Mentoring: Lernen aus Erfahrung? Biographisches Lernen im Kontext gesellschaftlicher Transformationsprozesse. Bielefeld: W. Bertelsmann Verlag.

Schulz, W. (1996). Lerngeschichten und Bildungswege. Anregungen und Reflexion zu biographischen Lernprozessen. Baltmannsweiler: Schneider Verlag Hohengehren.

Schulz, W. (2008). Lehrer werden - berufsbiografische Zugänge. Ein Werkstattbericht. In S. Kirchhof \& W. Schulz (Hrsg.), Biografisch lernen \& lehren. Möglichkeiten und Grenzen zur Entwicklung biografischer Kompetenz (S. 169-194). Flensburg: Flensburg University Press.

Siebert, H. (1985). Lernen im Lebenslauf. Zur biographischen Orientierung der Erwachsenenbildung. Frankfurt a. M.: Pädagogische Arbeitsstelle Deutscher Volkshochschul-Verband.

Wrana, D. (2006). Das Subjekt schreiben. Reflexive Praktiken und Subjektivierungen in der Weiterbildung. Eine Diskursanalyse. Baltmannsweiler: Schneider Verlag Hohengehren.

Zeuner, C., \& Faulstich, P. (2009). Erwachsenenbildung - Resultate der Forschung. Entwicklung, Situation und Perspektiven. Weinheim: Beltz.

\section{Bildungspolitische Dokumente}

Bundesministerium für Bildung und Forschung (BMBF). (2008). Konzeption der Bundesregierung zum Lernen im Lebenslauf. http://www.bmbf.de/de/lebenslangeslernen.php. Zugegriffen: 15. Nov. 2014.

Bundesministerium für Bildung und Forschung (BMBF). (2013). Weiterbildungsverhalten in Deutschland. AES 2012 Trendbericht. Bonn.

Bund-Länder-Kommission für Bildungsplanung und Forschungsförderung. (2004). Strategie für Lebenslanges Lernen in der Bundesrepublik. Materialien zur Bildungsplanung und Forschungsförderung, 115, Bonn. http://www.blk-bonn.de/papers/heft115.pdf. Zugegriffen am 23. Feb. 2015.

Vereinigung der bayrischen Wirtschaft. (2003). Bildung neu denken! Das Zukunftsprojekt. Opladen: Leske + Budrich. 